\title{
Confocal laser endomicroscopy detects colonic inflammation in patients with irritable bowel syndrome: a prospective study
}

\section{(ㄷ)(우우}

\begin{abstract}
Authors
\author{
Institution \\ Gastroenterology and Endoscopy Division, Instituto \\ Ecuatoriano de Enfermedades Digestivas (IECED), \\ Guayaquil, Ecuador
}

Carlos Robles-Medranda, Roberto Oleas, Manuel Valero, Miguel Puga-Tejada, Miguel Soria-Alcívar, Jesenia Ospina, Haydee Alvarado-Escobar, Guillermo Muñoz-Jurado, Jorge Baquerizo-Burgos, Hannah Pitanga-Lukashok (pCLE) and histological evaluation in IBS patients. submitted 19.7.2019

accepted after revision 20.12.2019

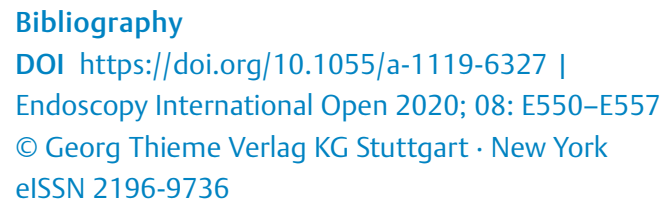

\section{Corresponding author}

Carlos Robles-Medranda, MD, Endoscopy Division, Instituto Ecuatoriano de Enfermedades Digestivas - IECED, Av. Abel Romeo Castillo y Av. Juan Tanca Marengo, Torre Vitalis, Mezanine 3, Guayaquil, Ecuador

Fax: +593-4-2109180

carlosoakm@yahoo.es

丹 Supplementary material

Online content viewable at:

https://doi.org/10.1055/a-1119-6327

\section{ABSTRACT}

Background and aims Irritable bowel syndrome (IBS) is considered to be a functional disease, but recent data indicate measurable organic alterations. We aimed to deter- mine the presence of colorectal mucosa microinflammation in vivo via probe-confocal laser endomicroscopy

Methods This was a prospective, controlled, nonrandomized single-blind diagnostic trial performed in a tertiary institution. PCLE images and targeted biopsy of each colon segment obtained during colonoscopies of IBS patients and controls were analyzed for inflammatory changes. Biopsies were classified using the Geboes scale, and the odds ratio and overall diagnostic accuracy were calculated.

Results During the 15 -month study period, 37 patients were allocated to each group. The mean age was $53.1 \pm$ 14.3 years; $64.9 \%$ were female. Signs of colonic mucosa inflammation were evident on $65.8 \%$ of pCLE images from IBS patients compared to $23.4 \%$ of images from controls (OR 6.28 ; 4.14-9.52; $P<0.001)$. In total, $20 / 37$ patients had microinflammation via $\mathrm{PCLE}$ in $\geq 3$ colon segments in the IBS group, compared to $1 / 37$ in the control group. A Geboes score $>0$ was attributed to $60.8 \%$ of biopsies from patients in the IBS group compared to $27.5 \%$ of biopsies from the control group. The sensitivity, specificity, positive and negative predictive values, observed and interrater agreement of pCLE-detected inflammatory changes in IBS using histology as gold standard were $76 \%, 91 \%, 76 \%, 91 \%, 86.5 \%$, and $66.8 \%$, respectively.

Conclusions Patients with IBS have a six-fold higher prevalence of colorectal mucosa microinflammation than healthy controls. pCLE might be a reliable method to detect colorectal mucosa microinflammation in IBS patients.

\section{Introduction}

Irritable bowel syndrome (IBS) is a clinical condition characterized by changes in bowel movement frequency and consistency and abdominal pain. IBS affects $10-20 \%$ of the population worldwide [1]. Currently, IBS diagnosis is based on the Rome IV criteria, derived from a consensus process by a multinational group of experts [2], which have a low sensitivity of $62.7 \%$ [3].
According to these criteria, IBS is diagnosed based on recurrent abdominal pain related to defecation or in association with a change in stool frequency or form. Based on the Rome IV criteria, IBS is classified into four subtypes according to patients' reports of the proportion of time they have hard or lumpy stools versus loose or watery stools [2].

IBS has traditionally been considered to be a chronic functional gastrointestinal disorder [4], as there are no reliable bio- 
markers or specific endoscopic findings that can discriminate affected patients from healthy controls $[5,6]$. Indeed, validation of the Rome IV diagnostic questionnaire for adults failed to produce high diagnostic sensitivity for an objective diagnosis of IBS in affected adults. The current modality test failed to produce an objective measured and evaluated indicator for the diagnosis of IBS [3].

Numerous studies have identified measurable components of IBS that suggest that it is an organic disorder. Altered gastrointestinal motility, visceral hypersensitivity, postinfectious reactivity, brain-gut interactions, altered fecal microflora, bacterial overgrowth, food sensitivity, carbohydrate malabsorption, and intestinal inflammation, however, have all been implicated in IBS pathogenesis [7]. Increased numbers of inflammatory cells and levels of cytokines in the colonic mucosa have also been detected in patients with IBS, especially those with diarrhea-predominant IBS and postinfectious IBS [7,8]. These findings support the hypothesis that immune activation may have a role in IBS pathogenesis, which has resulted in several studies exploring the use of inflammatory and immunological markers for the diagnosis of IBS. Specifically, impaired intestinal barrier function (gut permeability) and elevated inflammatory marker expression have been described and correlated with IBS severity [9]. Similarly, the quantification and distribution of colonic immune cells and immune activity have been studied by measuring cytokine levels and serum biomarker expression; however, these measures can only be evaluated using quantitative assessments [5, 10-13].

The mechanisms underlying IBS pathophysiology are complex and multifactorial. Several studies have suggested low grade colonic inflammation as an initiator of gut dysfunction in affected patients $[11,14]$. Other studies have shown an increase in the levels of inflammatory cytokines and oxidative stress serum biomarker levels in patients with IBS, suggesting a systematic inflammatory response [5,6]. A recent meta-analysis described an increase in the number of mucosal immune cells in colonic biopsies of patients with IBS, specifically mast cells and CD3 $+T$ cells [12], suggesting that low grade colonic inflammation plays a role in IBS pathophysiology $[5,6,12,13]$.

IBS is currently considered to be a diagnosis of exclusion; however, a positive diagnosis approach using an accurate diagnostic test is promising. White-light endoscopy can detect macroscopic features of inflammation, but its capacity to assess inflammation, identify specific findings and reliably diagnose IBS is low, and it is unable to detect specific signs of IBS [15]. Instead, endoscopy is performed to exclude other causes of the patients' symptoms.

Novel techniques, such as digital chromoendoscopy and optical magnification, cannot provide sufficient magnification to detect microinflammation. Probe-based confocal laser endomicroscopy (pCLE) combines real-time endoscopic and histologic evaluations with a high magnification (1000-fold magnification) [16]. Using this technique, endoscopists can visualize cellular details in vivo at a level comparable to that of conventional histologic slides. Furthermore, when fluorescein is used as a contrast agent, pCLE can detect the epithelial gap density of the intestinal epithelium, which is a marker of epithelial cell ex- trusion [17]. pCLE is, therefore, considered to be a reliable technique for in vivo, real-time assessment of intestinal barrier function and inflammatory activity [18]. In addition, pCLE provides improved mucosal analysis and biopsy targeting compared to conventional methods (colonoscopy with random biopsy samples) that yield a limited number of biopsies for histopathologic analysis, and have a risk of false negative results.

pCLE has been used in patients with IBS and suspected food intolerance. With this approach, increased epithelial gap density in the small bowel, immediate breaks, increased intervillous spaces, and increased intraepithelial lymphocytes in the mucosa have been associated with IBS $[17,19]$. There are currently no published reports detailing pCLE findings in the colonic mucosa of patients with IBS.

The aims of the present study were (1) to determine the presence of colorectal mucosa microinflammation in vivo via PCLE and histologic evaluation in patients with IBS, and (2) to determine whether pCLE is sufficiently sensitive and specific to differentiate IBS from healthy patients.

\section{Material and methods}

\section{Study design}

This prospective, controlled, nonrandomized and single-blind diagnostic trial was conducted at the Instituto Ecuatoriano de Enfermedades Digestivas (IECED) and registered at ClinicalTrials.gov under code NCT02651532. Patients were enrolled from January 2016 to March 2017.

The study protocol was reviewed and approved by the Institutional Review Board and Human Ethics Committee of our institution under protocol number ENE12016. The study was conducted in accordance with the Declaration of Helsinki. Informed and written consent was obtained from all participants. All authors had access to the study data and reviewed and approved the final manuscript.

\section{Population selection}

Consecutive patients $\geq 18$ years of age were invited to participate in the study and were assigned to one of two groups: the study group or the control group. The study group consisted of patients with an IBS diagnosis given within the past 12 months, based on the Rome IV criteria [3]. Recurrent abdominal pain, on average, at least 1 day/week in the last 3 months, associated with two or more of the following criteria: related to defecation, associated with a change in the frequency of stool, associated with a change in the form (appearance) of stool. The criteria must have been fulfilled for the last 3 months with symptom onset at least 6 months before diagnosis.

Symptoms had to be present $>25 \%$ of the time; symptoms were evaluated by means of daily diaries that were completed for 15 days and included a stool consistency rating using the Bristol stool form score [20]. The control group consisted of individuals without any digestive complaint but who were undergoing a colonoscopy exam most commonly for colorectal cancer screening. Control subjects had no macroscopic lesions visible by endoscopic examination, including polyps, ulcerations, or macroscopic signs of inflammation. 
All medical records, imaging data, and laboratory tests were reviewed by three gastroenterologists to exclude potential misdiagnoses.

\section{Inclusion criteria}

The inclusion criteria were as follows: inflammatory serum biomarkers (C-reactive protein and erythrocyte sedimentation rate) within normal limits, normal imaging tests (endoscopic and abdominal ultrasonography evaluation), negative serology for celiac disease, negative serial stool test examination for parasitic infection and culture, normal breath test in patients with suspected lactose intolerance or bacterial overgrowth, and a normal level of serum thyroid-stimulating hormone.

\section{Exclusion criteria}

Patients were excluded if they were pregnant or nursing, had a history of postinfectious IBS, lymphocytic or collagenous colitis, psychiatric comorbidities such as major depressive disorder or bipolar affective disorder, inflammatory bowel disease, colonic obstruction or resection, allergy to fluorescein, an inadequate quality of bowel preparation (Boston bowel preparation scale score $<2$ in any segment of the colon), or had been administered nonsteroidal anti-inflammatory drugs, acetylsalicylic acid, herbals, corticosteroids or antibiotics within the past 12 months. Included subjects denied smoking and alcohol consumption.

\section{Colonoscopy with pCLE}

All participants underwent colonoscopy examination performed by two endoscopists (CR-M, MV); both operators had completed training for $\mathrm{PCLE}$ image interpretation with a score $\geq$ 9 and had performed more than 100 pCLE procedures before the study. These operators were blinded to the patient medical records and group allocation. Bowel preparation was performed using polyethylene glycol-electrolyte solution for all participants. After deep sedation by intravenous propofol administration, a standard colonoscopy using a high definition scope with white light (Pentax EC-3890LZi, Tokyo, Japan) was performed. Care was given to avoid damaging the epithelium during mucosal inspection.

Patients were intravenously injected with $5 \mathrm{~mL}$ of $10 \%$ fluorescein (BioGlo, Sofar Productos, Bogota, Colombia), and then pCLE was performed in single endoscopic view in each anatomic colon segment using a ColoFlex probe (Cellvizio; Mauna Kea Technology, Paris, France) through the accessory channel of the scope. The probe was gently placed in contact with the mucosa to avoid trauma. Finally, a targeted biopsy of each colon segment evaluated by the pCLE probe was performed. A total of six biopsies per patient were obtained, one for each colonic segment (cecum, ascending colon, transverse colon, descending colon, sigmoid colon, and rectum). All mucosal biopsies were taken immediately after removal of the pCLE probe and as close as possible to the PCLE imaging site.

\section{pCLE inflammation criteria}

Altered crypt architecture (difference in shape, size, and distribution of crypts), increased epithelial gaps (increased distance between crypts) with fluorescein leaks into the crypt lumen, and dilated and prominent branching vessels detected by PCLE were considered to indicate inflammation [18].

\section{Correlation between pCLE and histological findings}

The Geboes scale was used to evaluate the histological aspects of the segments evaluated initially via pCLE [21]. The Geboes scale is composed of six grades: grade 0 exhibits structural changes, grade 1 chronic inflammation with cell infiltrate, grade 2 a lamina propria with neutrophils and eosinophils, grade 3 neutrophils in the epithelium, grade 4 crypt destruction, and grade 5 erosions or ulcers. All histologic samples were evaluated by a single pathologist who was blinded to the clinical and endoscopic information.

\section{Statistical analyses}

Sample size calculation

Using a $1 \%$ margin of error (type I and type II errors), we estimated that 37 participants in each group would be needed to detect inflammation in the colon of IBS patients. Prevalences of microinflammatory changes of $62.5 \%$ and $11.1 \%$ in the IBS and control groups, respectively, were estimated as previously described [17].

\section{Data analysis}

Continuous and categorical variables were compared by Student's $t$ test, Pearson chi-squared test or Fisher's exact test, as appropriate. The relationship between the study group and the presence of microinflammation on pCLE was determined using the odds ratio (OR) per colon segment. The overall diagnostic accuracy of PCLE for the diagnosis of IBS was determined by the sensitivity, specificity, positive predictive value (PPV), negative predictive value (NPV), and an off-line observed interrater agreement (Cohen's kappa). A $P$ value $<0.05$ was considered statistically significant. Data analysis was performed using $R$ version 3.4.3 (R Foundation for Statistical Computing, Vienna, Austria).

\section{Results}

We recruited 93 patients, of whom 37 were enrolled in the IBS group and 37 were enrolled in the control group; 19 patients were excluded across both groups due to inadequate bowel preparation, positive stool cultures or the presence of lesions during white light colonoscopy. The mean age of the IBS patients was $50.62 \pm 15.9$ years, and there were 29 (78.4\%) females, as IBS is more common in women. The mean age of the control patients was $55.51 \pm 12.3$ years, with 19 (51.44\%) females. The IBS group included 11 (29.7\%) cases of diarrheapredominant IBS, 21 (56.8\%) cases of constipation-predominant IBS and five $(13.5 \%)$ cases of alternating constipation and diarrhea IBS. A Boston Bowel Preparation Scale score of 8 (inter- 


\begin{tabular}{|c|c|c|c|c|}
\hline & Cases $(n=37)$ & Control $(n=37)$ & Total $(n=74)$ & $P$ value \\
\hline Cecum, n (\%) & $25(67.6)$ & $12(32.4)$ & $37(50.0)$ & 0.003 \\
\hline Ascending colon, n (\%) & $28(75.7)$ & $7(18.9)$ & $35(47.3)$ & $<0.001$ \\
\hline Transverse colon, n (\%) & $23(62.2)$ & $10(27.0)$ & $33(44.6)$ & 0.002 \\
\hline Descending colon, $\mathrm{n}(\%)$ & $18(48.6)$ & $6(16.2)$ & $24(32.4)$ & 0.003 \\
\hline Sigmoid colon, n (\%) & $26(70.3)$ & $8(21.6)$ & $34(45.9)$ & $<0.001$ \\
\hline Rectum, n (\%) & $26(70.3)$ & $9(24.3)$ & $35(47.3)$ & $<0.001$ \\
\hline Total & $146 / 222(65.8)$ & $52 / 222(23.4)$ & $198 / 444(44.6)$ & $<0.001$ \\
\hline
\end{tabular}

quartile range 6-9) was attributed to both the study group and control group. No adverse events occurred during the study.

\section{Inflammatory lesions detected during PCLE}

We detected a statistically significant difference in the number of inflammatory changes between cases and controls by PCLE and histological evaluation in all colon segments and the rectum. A significantly higher number of pCLE images with inflammatory lesions were obtained in the IBS group than in the control group (OR 6.28; 4.14-9.52; $P<0.001$ ). The percentage of pCLE images with inflammatory lesions detected in each colon segment was $65.8 \%$ in the IBS group. Twenty patients in the IBS group exhibited microinflammation via pCLE in three or more colon segments, which reflects a more severe clinical condition. In the control group, the percentage of pCLE images with inflammatory lesions detected in each colon segment was $23.4 \%$. One control subject exhibited microinflammation in three or more colon segments. In patients with IBS, most lesions occurred in the ascending colon, and in control subjects, most lesions occurred in the cecum. The inflammatory lesions found during PCLE in each colon segment are summarized in - Table 1. Subjects with a healthy colonic mucosa exhibited dark goblet cells, a round crypt structure with regular and narrow vessels surrounding the crypts. IBS patients exhibited cellular infiltration with fluorescein leaks into the crypt lumen; differences in the shapes, sizes, and distribution of the crypts; a large distance between crypts and focal crypt distribution; and mild-to-moderate increases in capillaries. Fig. 1 shows the endoscopic, pCLE, and histological findings of a patient with constipation-predominant IBS. These data indicate that organic alterations in patients with IBS can be detected by pCLE and open a discussion about the functional versus organic nature of IBS.

\section{pCLE target biopsy analysis}

All mucosal biopsies were taken immediately after removal of the pCLE probe and as close as possible to the pCLE imaging site. We obtained 444 biopsies from the 74 patients included in the study. Pathology results indicated that one patient from the IBS group had eosinophilic colitis, and no patient met the
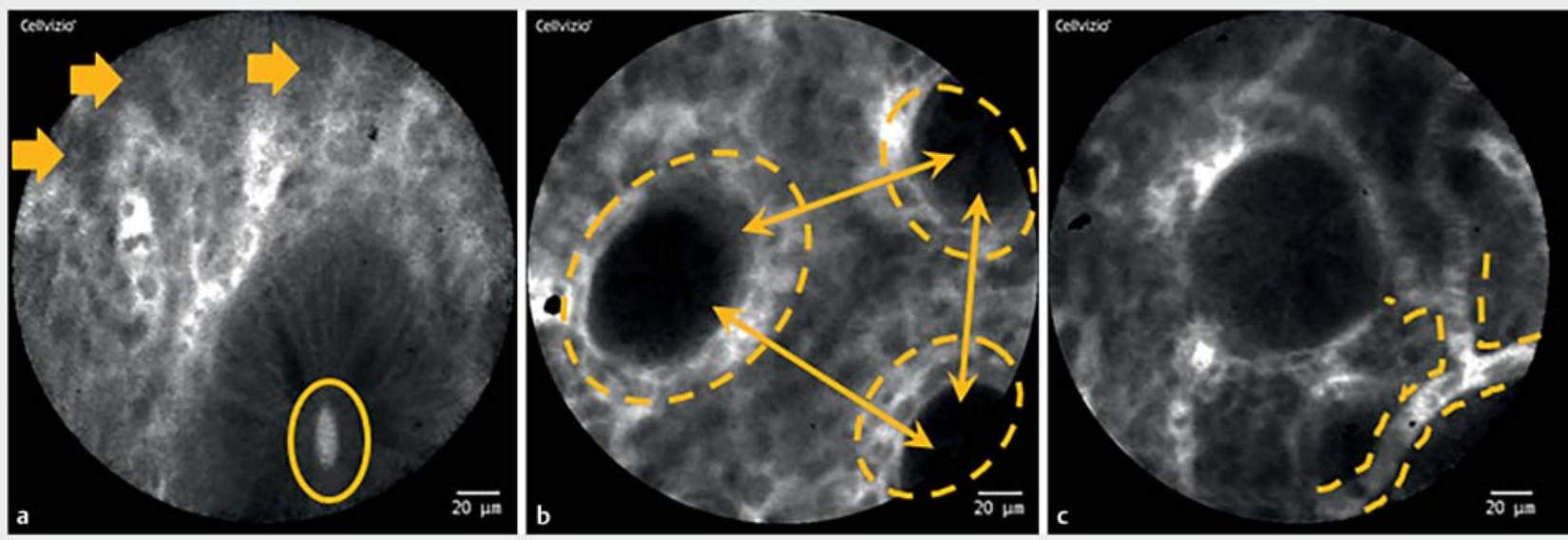

- Fig. 1 pCLE inflammation criteria. a Cellular infiltration present (arrows), fluorescein leaks into the crypt lumen (ellipse). b Differences in shapes, sizes and distribution of crypts (ellipses); increased distance between crypts, focal crypt distribution (arrows). c Mild to moderate increase in capillaries, dilated and distorted capillaries (dashed lines). 
- Table 2 Number of colonic segments with inflammatory lesions detected during histological evaluation according to the Geboes scale (>0).

\begin{tabular}{|l|c|c|c|}
\hline & Cases $(\mathbf{n}=\mathbf{3 7})$ & Control (n=37) & 0.005 \\
\hline Cecum, $\mathrm{n}(\%)$ & $26(70.27)$ & $14(37.83)$ & $<0.001$ \\
\hline Ascending colon, $\mathrm{n}(\%)$ & $28(75.67)$ & $11(29.72)$ & 0.036 \\
\hline Transverse colon, $\mathrm{n}(\%)$ & $22(59.45)$ & $13(35.13)$ & 0.079 \\
\hline Descending colon, $\mathrm{n}(\%)$ & $15(40.54)$ & $8(21.62)$ & $<001$ \\
\hline Sigmoid colon, $\mathrm{n}(\%)$ & $26(70.27)$ & $8(21.62)$ & 0.007 \\
\hline Rectum, $\mathrm{n}(\%)$ & $18(48.64)$ & $7(18.91)$ & $<0.001$ \\
\hline Total & $135 / 222(60.8)$ & $61 / 222(27.5)$ & \\
\hline
\end{tabular}

criteria for either ulcerative colitis or lymphocytic colitis. In a sub-analysis of cell infiltrate among different IBS phenotypes (37 IBS patients, 11/37 diarrhea-predominant IBS, 21/37 constipation-predominant IBS, and 5/37 alternating IBS), we found no statistically significant difference (Supplementary table 1).

\section{Histologic findings by the Geboes scale}

Next, the histological findings were assigned a score according to the Geboes scale in all evaluated segments. Here, we found that a Geboes score $>0$ was more frequently found in the IBS group than in the control group $(60.8 \%$ vs. $27.5 \%$; $P<0.001)$ ( $\triangleright$ Table 2). Eighteen IBS patients exhibited a Geboes score $>0$ in three or more colon segments. Three control subjects exhibited a Geboes score $>0$ in three or more colon segments. We found a statistically significant difference in the distribution of the Geboes scores between cases and control groups in the cecum, ascending colon, sigmoid colon, and rectum expressed by the number of patients $(P=0.048, P=0.003, P=0.001$, and $P=$ 0.046 , respectively). $>$ Fig. 2 represents the distribution of the assigned Geboes score in each study group expressed by the number of patients and colon segment. However, in a sub-analysis of PCLE inflammatory findings among each Geboes score in the IBS and control group, we found no statistically significant differences (Supplementary table 2).

\section{Overall diagnostic accuracy of the PCLE inflammatory findings}

When considering a target biopsy of a segment as the gold standard, the inflammatory findings detected during PCLE colonoscopy in patients with IBS showed a sensitivity, specificity, PPV, NPV, and observed and interrater agreement of $86.7 \%, 88.7 \%, 85.9 \%, 89.4 \%, 87.8 \%$, and $75.4 \%$, respectively ( $\triangleright$ Table 3$)$. There were no statistically significant differences in the pooled sensitivity $(P=0.4680)$ or specificity $(P=0.1427)$ for each colon segment or the rectum. - Table 4 shows the overall diagnostic accuracy of the pCLE inflammatory findings per IBS patient; biopsies were considered positive for inflammation when the Geboes score was greater than zero. An individual was considered positive for inflammation when more than three of the six biopsies had a Geboes score $>0$.

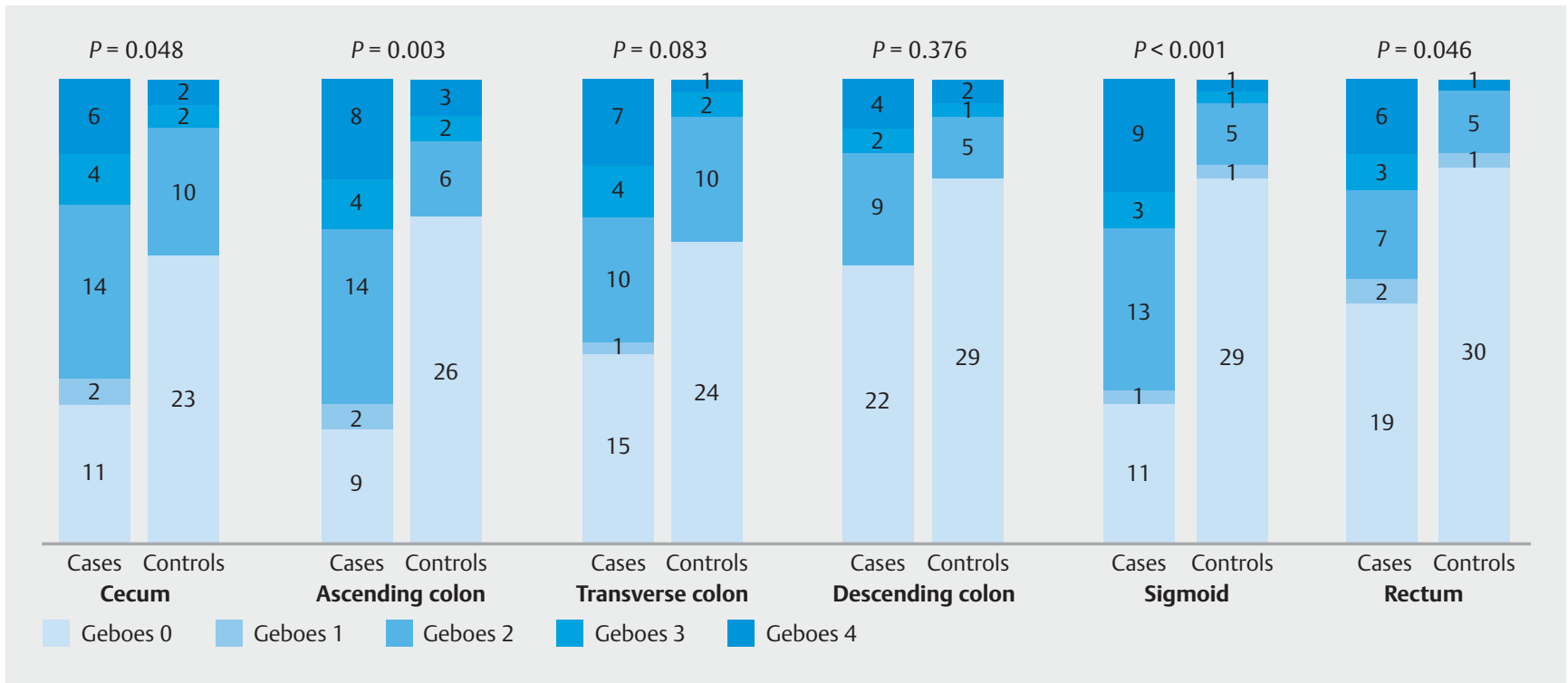

Fig. 2 Histological findings compatible with inflammation in accordance with the Geboes scale in each colorectal segment. 
- Table 3 Overall diagnostic accuracy of pCLE in detecting inflammatory changes in the 37 patients with IBS when considering target biopsy as the gold standard.

\begin{tabular}{|c|c|c|c|c|c|c|c|}
\hline & Cecum & $\begin{array}{l}\text { Ascending } \\
\text { colon }\end{array}$ & $\begin{array}{l}\text { Transverse } \\
\text { colon }\end{array}$ & $\begin{array}{l}\text { Descending } \\
\text { colon }\end{array}$ & $\begin{array}{l}\text { Sigmoid } \\
\text { colon }\end{array}$ & Rectum & Total \\
\hline $\begin{array}{l}\text { Sensitivity, \% } \\
\text { (range) }\end{array}$ & $\begin{array}{l}87.5 \\
(73.2-95.8)\end{array}$ & $\begin{array}{l}82.1 \\
(66.5-92.5)\end{array}$ & $\begin{array}{l}88.6 \\
(73.3-96.8)\end{array}$ & $\begin{array}{l}78.3 \\
(56.3-92.5)\end{array}$ & $\begin{array}{l}88.2 \\
(72.6-96.7)\end{array}$ & $\begin{array}{l}96.0 \\
(79.7-99.9)\end{array}$ & $\begin{array}{l}86.7 \\
(81.2-91,2)\end{array}$ \\
\hline $\begin{array}{l}\text { Specificity, \% } \\
\text { (range) }\end{array}$ & $\begin{array}{l}94.1 \\
(80.3-99.3)\end{array}$ & $\begin{array}{l}91.4 \\
(76.9-98.2)\end{array}$ & $\begin{array}{l}94.9 \\
(82.7-99.4)\end{array}$ & $\begin{array}{l}88.2 \\
(76.1-5.6)\end{array}$ & $\begin{array}{l}90.0 \\
(76.3-97.2)\end{array}$ & $\begin{array}{l}77.6 \\
(63.4-88.2)\end{array}$ & $\begin{array}{l}88.7 \\
(84.1-92.4)\end{array}$ \\
\hline PPV, \% (range) & $\begin{array}{l}94.6 \\
(81.9-98.5)\end{array}$ & $\begin{array}{l}91.4 \\
(78.2-97.0)\end{array}$ & $\begin{array}{l}93.9 \\
(80.0-98.4)\end{array}$ & $\begin{array}{l}75.0 \\
(59.7-86.2)\end{array}$ & $\begin{array}{l}88.2 \\
(76.4-95.0)\end{array}$ & $\begin{array}{l}68.6 \\
(56.3-78.7)\end{array}$ & $\begin{array}{l}85.9 \\
(81.0-89.6)\end{array}$ \\
\hline NPV, \% (range) & $\begin{array}{l}86.5 \\
(73.7-93.6)\end{array}$ & $\begin{array}{l}82.1 \\
(69.9-90.0)\end{array}$ & $\begin{array}{l}90.2 \\
(78.6-95.9)\end{array}$ & $\begin{array}{l}90.0 \\
(80.5-95.2)\end{array}$ & $\begin{array}{l}90.0 \\
(78.1-95.8)\end{array}$ & $\begin{array}{l}97.4 \\
(84.7-99.6)\end{array}$ & $\begin{array}{l}89.4 \\
(85.5-92.4)\end{array}$ \\
\hline $\begin{array}{l}\text { Observed } \\
\text { agreement }\end{array}$ & 90.5 & 86.5 & 91.9 & 91.9 & 89.2 & 83.8 & 87.8 \\
\hline $\begin{array}{l}\text { Inter-rater } \\
\text { agreement }\end{array}$ & $\begin{array}{l}81.1 \\
(67.8-94.4)\end{array}$ & $\begin{array}{l}73.1 \\
(57.6-88.5)\end{array}$ & $\begin{array}{l}83.7 \\
(71.2-96.2)\end{array}$ & $\begin{array}{l}65.7 \\
(47.2-84.2)\end{array}$ & $\begin{array}{l}78.2 \\
(64.0-92.5)\end{array}$ & $\begin{array}{l}67.0 \\
(50.6-83.4)\end{array}$ & $\begin{array}{l}75.4 \\
(69.2-81.5)\end{array}$ \\
\hline
\end{tabular}

IBS, irritable bowel syndrome; PCLE, probe-confocal laser endomicroscopy; PPV, positive predictive value; NPV, negative predictive value.

- Table 4 Overall diagnostic accuracy of pCLE for the inflammatory findings in the IBS group $(n=37)$ per patient when considering $p$-CLE target biopsy as the gold standard $(\%, 95 \% \mathrm{Cl})$.

\begin{tabular}{|c|c|c|c|c|c|c|c|}
\hline & Cecum ${ }^{1}$ & Right colon ${ }^{1}$ & $\begin{array}{l}\text { Transverse } \\
\text { colon }^{1}\end{array}$ & Left colon ${ }^{1}$ & Sigmoid ${ }^{1}$ & Rectum ${ }^{1}$ & Total $^{2}$ \\
\hline Sensitivity & $88(73-96)$ & $82(66-92)$ & $89(73-97)$ & $78(56-93)$ & $88(73-97)$ & $96(80-100)$ & $76(53-92)$ \\
\hline Specificity & 94 (80-99) & $91(77-98)$ & 95 (83-99) & $88(76-96)$ & 90 (76-97) & $78(63-88)$ & 91 (79-97) \\
\hline PPV & 95 (82-99) & $91(77-98)$ & 94 (80-99) & $75(53-90)$ & $88(73-97)$ & $69(51-83)$ & $76(53-92)$ \\
\hline NPV & $86(71-95)$ & $82(66-92)$ & 90 (77-97) & $90(78-97)$ & 90 (76-97) & $97(87-100)$ & 91 (79-97) \\
\hline $\begin{array}{l}\text { Observed } \\
\text { agreement }\end{array}$ & $90.5 \%$ & $86.4 \%$ & $91.9 \%$ & $85.1 \%$ & $89.1 \%$ & $83.7 \%$ & $86.5 \%$ \\
\hline $\begin{array}{l}\text { Inter-rater } \\
\text { agreement }\end{array}$ & $\begin{array}{l}81.1 \% \\
(P<0.001)\end{array}$ & $\begin{array}{l}73.1 \% \\
(P<0.001)\end{array}$ & $\begin{array}{l}83.7 \% \\
(P<0.001)\end{array}$ & $\begin{array}{l}65.7 \% \\
(P<0.001)\end{array}$ & $\begin{array}{l}78.2 \% \\
(P<0.001)\end{array}$ & $\begin{array}{l}67.0 \% \\
(P<0.001)\end{array}$ & $\begin{array}{l}66.8 \% \\
(P<0.001)\end{array}$ \\
\hline
\end{tabular}

\section{Discussion}

We aimed to determine the presence of colorectal mucosa microinflammation in vivo via pCLE and histological evaluation in patients with IBS and to determine whether PCLE is sufficiently sensitive and specific to differentiate IBS from healthy patients. In the present study, we found that PCLE and histologic signs of microinflammation, such as an altered crypt architecture, increased epithelial gaps with fluorescein leaks, and dilated and prominent branching vessels, are sufficiently sensitive and specific for differentiating healthy individuals from patients with IBS.

Compared to the control group, we detected a significant increase in the number of pCLE inflammatory findings in patients with IBS, equating to an approximately six-fold higher prevalence of microinflammation compared to healthy individuals
(OR 6.28; 4.14-9.52; $P<0.001$ ). The ascending and sigmoid colons and the rectum exhibited the greatest number of inflammatory lesions. Inflammatory changes have mainly been reported in patients with diarrhea-predominant IBS and postinfectious IBS and have been detected in the small bowel [17]. Interestingly, we detected inflammatory changes across all IBS subtypes included in our cohort $(56.8 \%$ had constipation-predominant IBS). To the best of our knowledge, our study might be the first to use $\mathrm{pCLE}$ to evaluate microinflammation markers in vivo in the colon of patients with IBS.

IBS diagnosis is currently based on the Rome IV criteria [3, 4, 7], and neither endoscopic nor histologic markers can improve IBS diagnostic accuracy. Some have proposed that inflammatory cell distribution in the colon mucosa may be useful to facilitate the diagnosis of IBS [12]; however, one study found no difference in global cellularity in colonic biopsies of four distinct 
regions of the colon between 14 IBS patients and 20 control subjects [10]. Our study has shown that IBS patients have a higher prevalence of colorectal mucosa microinflammation detected via PCLE and histological evaluation, with an improvement in sensitivity over the IV Rome criteria questionnaire. However, a pilot study about the distribution of inflammatory cells in the colon was not able to detect a difference in the global cellularity between IBS patients and control subjects [10].

In the present study, our PCLE analysis of all colonic segments (using the proposed inflammatory criteria for the diagnosis of microinflammation in patients with IBS) had a sensitivity of $86.7 \%$ and a specificity of $88.7 \%$ using histopathological analysis of the inflammation as the gold standard.

The cost of performing a pCLE evaluation of the colon during colonoscopy is $\$ 375.00$, with an increase in procedure time of approximately 10 minutes. Moreover, the implementation of pCLE during colonoscopy might play a role in a positive diagnostic strategy for IBS, thus avoiding making a diagnosis of IBS solely after the exclusion of other medical conditions. Furthermore, current protocols of random biopsies of the colon (four biopsies every four quadrants) in patients with inflammatory bowel disease are inferior compared to PCLE targeted biopsies for the detection of intraepithelial neoplasia [22]. In addition, pCLE has the advantage of being cost-effective compared to random biopsies, considering that digital biopsy via PCLE is cost-effective when more than 10 biopsies are indicated in the patient [23].

Our study has the advantage of detecting microinflammation via PCLE in the colorectal mucosa of patients with IBS, a prevalent condition worldwide. Previous studies describing low grade inflammation used expensive, quantitative, and time-consuming methods such as counting the number and distribution of inflammatory cells and the number of cytokines in the colonic mucosa. However, pCLE offers a real-time evaluation of the colonic mucosa with a reliable detection of low grade inflammation, as confirmed by the histological results in this study. Our study is limited by the small IBS population and its single-center nature. Although the Geboes score was developed for judging ulcerative colitis inflammation, we used this score to assess inflammation in the colonic mucosa of patients with IBS. In addition, an objective measurement of IBS severity using validated scales was not carried out in our study. Even with these constraints, all possible misdiagnoses were excluded before pCLE and histopathological analysis, and our population was adequately representative and included different IBS subtypes.

The present study showed the potential benefit of pCLE for identifying signs of microinflammation in each segment of the colon in patients with IBS in addition to histopathological deviations and opens a discussion about evaluating other specific treatments, such as budesonide and mesalazine, for IBS patients with documented low grade microinflammation [24]. In addition, the higher prevalence of low grade inflammation in the colonic mucosa opens a discussion about the role of low grade microinflammation in the pathogenesis of IBS and the rationale for anti-inflammatory drugs for the management of IBS.
Further prospective multicenter studies with larger cohorts are now necessary to validate and ensure the utility of pCLE in mapping inflammation in the colonic mucosa in patients with IBS. Additionally, it would be interesting to evaluate pCLE inflammatory patterns by IBS subtypes and IBS severity of symptoms and determine the stage after initial symptom onset at which PCLE can accurately detect inflammation in IBS patients.

In conclusion, pCLE proved to be a reliable method for detecting colorectal mucosa microinflammation in patients with IBS, showing that patients with IBS have a six-fold higher prevalence of colorectal mucosa microinflammatory features than healthy patients. pCLE evaluation of the colon is an accurate diagnostic test for IBS microinflammation detection and could be considered in a positive diagnostic approach. We hope that this study will encourage research in the field of microinflammation in the colons of patients with IBS.

\section{Competing interests}

The authors declare that they have no conflicts of interest.

\section{Clinical trial}

\section{Clinical.Trials.gov}

NCT02651535

TRIAL REGISTRATION: This was a prospective, controlled, nonrandomized and single-blind diagnostic trial registered under the code NCT02651535 at clinicaltrials.gov

\section{References}

[1] Longstreth GF, Thompson WG, Chey WD et al. Functional bowel disorders. Gastroenterology 2006; 130: 1480-1491

[2] Lacy BE, Mearin F, Chang L et al. Bowel disorders. Gastroenterology 2016; 150: 1393-1407

[3] Palsson OS, Whitehead WE, van Tilburg MAL et al. Development and validation of the Rome IV Diagnostic Questionnaire for Adults. Gastroenterology 2016; 150: 1481-1491

[4] Drossman DA. The functional gastrointestinal disorders and the Rome III process. Gastroenterology 2006; 130: 1377-1390

[5] Choghakhori R, Abbasnezhad A, Hasanvand A et al. Inflammatory cytokines and oxidative stress biomarkers in irritable bowel syndrome: Association with digestive symptoms and quality of life. Cytokine 2017; 93: 34-43

[6] Seyedmirzaee S, Hayatbakhsh MM, Ahmadi B et al. Serum immune biomarkers in irritable bowel syndrome. Clin Res Hepatol Gastroenterol 2016; 40: 631-637

[7] Saha L. Irritable bowel syndrome: Pathogenesis, diagnosis, treatment, and evidence-based medicine. World J Gastroenterol 2014; 20: 6759

[8] Spiller R, Garsed K. Postinfectious irritable bowel syndrome. Gastroenterology 2009; 136: 1979-1988

[9] Shulman RJ, Jarrett ME, Cain KC et al. Associations among gut permeability, inflammatory markers, and symptoms in patients with irritable bowel syndrome. J Gastroenterol 2014; 49: 1467-1476

[10] Boyer ], Saint-Paul M-C, Dadone B et al. Inflammatory cell distribution in colon mucosa as a new tool for diagnosis of irritable bowel syn- 
drome: A promising pilot study. Neurogastroenterol Motil 2018; 30: e13223

[11] Ford AC, Talley NJ. Mucosal inflammation as a potential etiological factor in irritable bowel syndrome: a systematic review. J Gastroenterol 2011; 46: 421-431

[12] Bashashati M, Moossavi S, Cremon C et al. Colonic immune cells in irritable bowel syndrome: A systematic review and meta-analysis. Neurogastroenterol Motil 2018; 30: e13192

[13] Ohman L, Isaksson S, Lundgren A et al. A controlled study of colonic immune activity and beta7+ blood T lymphocytes in patients with irritable bowel syndrome. Clin Gastroenterol Hepatol 2005; 3: 980-986

[14] Barbara G, De Giorgio R, Stanghellini V et al. A role for inflammation in irritable bowel syndrome? Gut 2002; 51: (Suppl. 01): 41-44

[15] Chey WD, Nojkov B, Rubenstein JH et al. The yield of colonoscopy in patients with non-constipated irritable bowel syndrome: results from a prospective, controlled US trial. Am J Gastroenterol 2010; 105: 859865

[16] Robles-Medranda C, Vargas M, Ospina J et al. Clinical impact of confocal laser endomicroscopy in the management of gastrointestinal lesions with an uncertain diagnosis. World J Gastrointest Endosc 2017; 16: 389-395

[17] Turcotte J-F, Kao D, Mah S] et al. Breaks in the wall: increased gaps in the intestinal epithelium of irritable bowel syndrome patients identi- fied by confocal laser endomicroscopy (with videos). Gastrointest Endosc 2013; 77: 624-630

[18] Li C-Q, Xie X-J, Yu T et al. Classification of inflammation activity in ulcerative colitis by confocal laser endomicroscopy. Am J Gastroenterol 2010; 105: 1391-1396

[19] Fritscher-Ravens A, Schuppan D, Ellrichmann M et al. Confocal endomicroscopy shows food-associated changes in the intestinal mucosa of patients with irritable bowel syndrome. Gastroenterology 2014; 147: $1012-1020$

[20] Lewis S], Heaton KW. Stool form scale as a useful guide to intestinal transit time. Scand J Gastroenterol 1997; 32: 920-924

[21] Geboes K, Riddell R, Ost A et al. A reproducible grading scale for histological assessment of inflammation in ulcerative colitis. Gut 2000; 47: 404-409

[22] Günther U, Kusch D, Heller F et al. Surveillance colonoscopy in patients with inflammatory bowel disease: comparison of random biopsy vs. targeted biopsy protocols. Int J Colorectal Dis 2011; 26 : 667-672

[23] Robles-Medranda C, Valero V, Puga-Tejada M et al. Mo1992 cost-effective analysis comparing standard biopsy vs. digital biopsy by confocal endomicroscopy. Gastrointest Endosc 2017; 85: 05AB510AB5 11

[24] Barbara G, Cremon C, Annese V et al. Randomised controlled trial of mesalazine in IBS. Gut 2016; 65: 82-90 